\title{
Cognitive ergonomics for data analysis
}

\author{
Experimental study of cognitive limitations in a data-based judgement task
}

\author{
Virpi Kalakoski \\ Finnish Institute of Occupational \\ Health \\ Helsinki, Finland, \\ virpi.kalakoski@ttl.fi
}

\author{
Andreas Henelius \\ Department of Computer Science \\ University of Helsinki, Helsinki, \\ Finland, \\ andreas.henelius@helsinki.fi
}

\author{
Emilia Oikarinen \\ Department of Computer Science \\ University of Helsinki, Helsinki, \\ Finland, \\ emilia.oikarinen@helsinki.fi
}

\author{
Antti Ukkonen \\ Department of Computer Science \\ University of Helsinki, Helsinki, \\ Finland, antti.ukkonen@helsinki.fi
}

\author{
Kai Puolamäki \\ Department of Computer Science \\ University of Helsinki, Helsinki, \\ Finland, kai.puolamaki@helsinki.fi
}

\begin{abstract}
Today's ever-increasing amount of data places new demands on cognitive ergonomics and requires new design ideas to ensure successful human-data interaction. Our aim is to identify the cognitive factors that require attention when designing systems to improve decision-making based on large amounts of data. We designed an experiment that simulates the typical cognitive demands people encounter in data analysis situations. We demonstrate some essential cognitive limitations using a behavioural experiment with 20 participants. The studied task presented the participants with critical and noncritical attributes that contained information on two groups of people. They had to select the response option (group) with a higher frequency of critical attributes. The results showed that accuracy of judgement decreased as the amount of information increased, and that judgement was affected by irrelevant information. Our results thus demonstrate critical cognitive limitations when people utilise data and suggest a cognitive bias in data-based decision-making. Therefore, when designing for cognition, we should consider the human cognitive limitations that are manifested in a data analysis context and develop general cognitive ergonomics guidelines for design to support the utilisation of data and improve data-based decision-making.
\end{abstract}

\section{CCS CONCEPTS}

- Human-centered computing Laboratory experiments • Human-centered computing Empirical studies in HCI • Applied computing Psychology

\section{KEYWORDS}

Human-data interaction, Decision-making, Cognitive ergonomics

Permission to make digital or hard copies of part or all of this work for personal or classroom use is granted without fee provided that copies are not made or distributed for profit or commercial advantage and that copies bear this notice and the full citation on the first page. Copyrights for third-party components of this work must be honored. For all other uses, contact the Owner/Author.

\section{ACM Reference format:}

Virpi Kalakoski, Andreas Henelius, Emilia Oikarinen, Antti Ukkonen and Kai Puolamäki. 2019. Cognitive ergonomics for data analysis: Experimental study of cognitive limitations in a data-based judgement task. In Proceedings of 31st European Conference on Cognitive Ergonomics (ECCE 2019), September 10-13, 2019, BELFAST, United Kingdom. ACM, New York, NY, USA, 5 pages. https://doi.org/10.1145/3335082.3335112

\section{Introduction}

In this era of digitalisation, humans need to increasingly make decisions that rely on data analysed and summarised by algorithms and systems. Data-based decision-making may appear simple on the surface: systems such as search engines provide the most important parts of the data for the experts and laymen interacting with the system to enable them to make rational decisions based on the evidence presented. However, previous research on human engineering and decision-making has already demonstrated that human-computer interaction is not always effortless and human decisions are prone to errors $[1,2]$. The cognitive ergonomics approach [3] and findings regarding experimental and applied cognitive psychology in human cognitive function [4] are essential to ensure successful 'humandata' interaction when designing systems and practices for the increasing number of contexts in which people utilise big data.

Two major limitations need to be considered. First, the amount of data has increased and the methods to analyse them have advanced considerably in recent years, but the basic cognitive ability of human beings has not developed in the same way. On one hand, human decision-making is still limited by, for example, our capacity to rehearse and process information in short-term working memory $[5,6]$, our inclination to better recall the first and last items from serially presented information [7], and the

ECCE 2019, September 10-13, 2019, BELFAST, United Kingdom (C) 2019 Copyright is held by the owner/author(s). ACM ISBN 978-1-4503-7166-7/19/09.

https://doi.org/10.1145/3335082.3335112 
various cognitive tendencies that bias our decisions [8]. On the other hand, although the human ability to learn is exceptional, developing expert-level knowledge and skills is time-consuming [9], and we seldom acquire exceptional or even adequate skill levels in a wide range of domains. For example, domain experts are rarely experts in data analysis, and they often have little knowhow or control of the systems used to analyse and summarise their data.

Second, although advances in computation and data analysis make it possible to differentiate complex patterns from large heterogeneous data sets, a query may appear fundamentally different when parameters or filtering criteria are changed. As a result, we live in 'filter bubbles' created by information processing systems [10], and large parts of important, significant, or novel views of the data may remain unnoticed. Therefore, despite the availability of huge amounts of data, we are not able to thoroughly utilise the information and evidence present in them. Our decisions may hence be founded on narrow views that exclude the critical factors necessary for making good, informed decisions [2].

The aim of this paper is to identify the cognitive factors that need attention when designing systems that support decisionmaking in contexts in which large data sets are utilised to improve evidence-based management [11, 12]. First, the amount of information required in everyday decision-making easily exceeds the limited capacity of working memory [6]. This cognitive limitation constrains how humans represent and rehearse the essential features and parameters of the data, and how much of this information is used when making decisions and judgements [13]. Second, when presenting data, it is seldom possible to present all the information simultaneously. Information is therefore often presented sequentially in smaller pieces. Research shows that the position of information affects encoding and the memory of information and decision-making [7, 14, 15]. Third, data-based decision-making is also a specific example of a demanding judgment task and thus likely to be subject to the biases typical to human thinking, such as our tendency to search for evidence matching our expectations (confirmation bias) [16], and the 'framing' of information affecting our choices [7, 15, 17]. In our study, we systematically approach these cognitive demands and limitations and investigate how the amount of presented information and cognitive biases manifest in data-based decisionmaking.

\section{Methods}

\subsection{Participants}

The participants were 20 volunteers (14 of them female) aged between 18 and 49 .

\subsection{Material}

A new judgement task was created for the experiment. The stimuli consisted of three critical target attributes and three noncritical background attributes associated with numbers between 1 and 10, which described the level of prevalence of the attribute in Group A and Group B. Participants were instructed to determine whether Group A or Group B had a higher workload, based on the values for the presented critical attributes only. The critical target attributes were related to workload and referred to disruptions, time pressure, and information overload, whereas the noncritical background attributes were neutral or related to the alleviation of workload and referred to team work, breaks and learning. The stimuli were assembled as sequences including one, two, or three critical and noncritical attributes (Figure 1). The sequences belonged to either of two ambiguity categories, depending on the total accumulated score for the critical target attributes in the sequence. In an unambiguous sequence, the total accumulated critical attribute in the sequence was greater for either Group A or B, that is, it was clear whether Group A or B had a higher workload. In an ambiguous sequence, the total accumulated critical attribute score was the same for both groups, that is, both choices were equally valid. The total accumulated score for the background attributes was always unambiguous and thus either supported or did not support either Group A or B. Each participant had altogether 192 sequences. A detailed description of how the ambiguous and unambiguous sequences and stimulus sets were constructed is available on request.

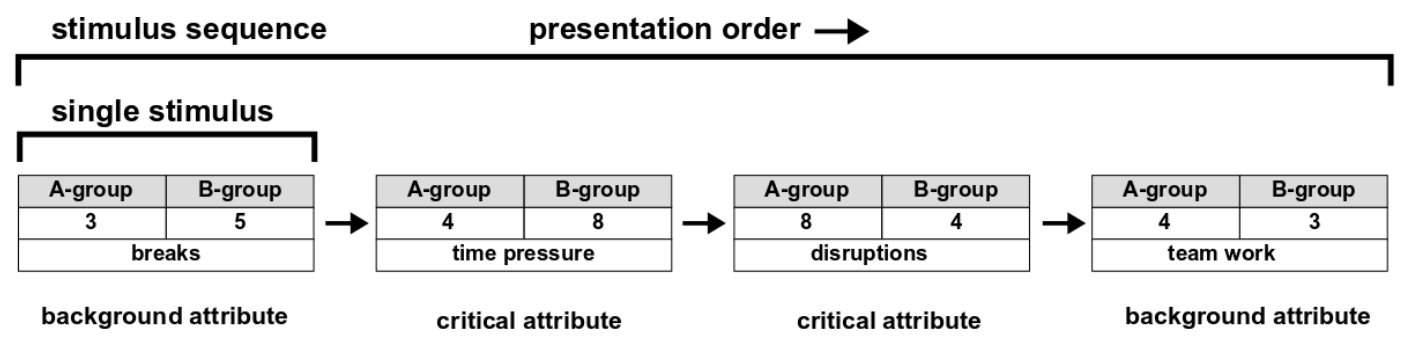

Figure 1: Example of an ambiguous stimulus sequence with four single stimuli. The actual stimuli were in Finnish. 


\section{Procedure}

The stimulus sequences were presented to the participants on a computer screen. Before each stimulus, a fixation dot was shown for $500 \mathrm{~ms}$, after which the stimulus was shown for $3000 \mathrm{~ms}$. After the last stimulus in the sequence, the response screen was visible until the participant pressed one of the reply keys, indicating Group A or B, or for a maximum time of $5000 \mathrm{~ms}$. The participant's selection was highlighted for $500 \mathrm{~ms}$. After this, the process was repeated for the next stimulus sequence.

\section{Results}

The independent variables were (i) the amount of presented information (length of a stimulus sequence, that is, the number of items within a sequence), (ii) the difficulty of the decision-making task (unambiguous vs ambiguous stimulus sets), and (iii) the role of background information (values for stimulus attributes defined as information irrelevant to the task). The dependent variables were the mean response time and the percentage of correct replies.

The percentages of correct responses showed that, as the amount of information increased, the level of correct responses decreased. Furthermore, the level of correct responses was higher and the response times were shorter when the noncritical background information supported rather than conflicted with the correct response based on the critical target information. Moreover, the distribution of different response categories showed that if the task had no correct reply (ambiguous sequences) but the background features supported either of the response options (A or $\mathrm{B}$ ), the participants used this response category more often than expected.

\section{Discussion}

In our study, we focused on the cognitive factors relevant to making judgements in data-based decision-making tasks. It demonstrates some well-known cognitive limitations in this context. The main findings were that 1) the response accuracy in data-based decision-making decreases when the amount of information increases and 2) noncritical background information is used in judgement. These results are in line with the vast literature demonstrating the limitations of cognitive capacity when the ongoing task requires maintaining and processing information in the working memory [6], as well as with research demonstrating cognitive biases in decision-making, such as judgement being framed by inessential information [2]. The contribution of our study is that we demonstrated several kinds of cognitive limitations that together constrain the utilisation of data. Therefore, when designing human-data interaction, we need to draw understanding from several fields of cognition and from multiple theoretical frameworks if we wish to compensate for the limited human cognitive capacity impairing our ability to efficiently utilise data.

We also need to develop good evidence-based cognitive ergonomics principles in design, which improve the quality of data-based decisions. These principles should at least (i) support the encoding of the reality of the situation by, for example, shortening the sequences when presenting information; (ii) enhance representation construction if several stages and subprocesses are required when presenting the data using, for example, a clever sequential presentation system; (iii) support ways of communicating which aspects of the data are considered meaningful to the user in that context; and (iv) make the human decision-maker aware of incidental attributes that may frame their decisions and cause them to lean towards unimportant aspects of the data. Furthermore, it would be useful to utilise data on human behaviour while the user is analysing a data set, in the same way as search engines adapt to the user when they navigate the internet. For example, identifying 'outliers' in response patterns in data-usage behaviour would allow the system to immediately recognise when the human decision-maker needs more support - or when there is a risk of cognitive bias - to improve the quality of their decisions.

\section{ACKNOWLEDGMENTS}

This study was supported by the Academy of Finland (decisions 326280 and 326339). We are thankful to Mr. Tomi Passi for his help in recruiting the participants and running the experiments.

\section{REFERENCES}

[1] CD Wickens, JD Lee; Y Liu, and SE Gorden-Becker (2003). An introduction to human factors engineering (2nd. ed.). Prentice Hall.

[2] H Judith, E Hibbard, and E Peters (2003). Supporting Informed Consumer Health Care Decisions: Data Presentation Approaches that Facilitate the Use of Information in Choice. Annual Review of Public Health 24, 413-433.

[3] IEA - International Ergonomics Association. Retrieved on 1 February 2019, https://www.iea.cc/

[4] V Kalakoski (2016). Cognitive Ergonomics. Retrieved on 1 February 2019, https://oshwiki.eu/index.php?title=Cognitive_ergonomics\&oldid=245803.

[5] AD Baddeley and G Hitch (1974). Working memory. The Psychology of Learning and Motivation, 8, 47-89. New York: Academic Press.

[6] N Cowan (2001). The Magical Number 4 in Short-Term Memory: A Reconsideration of Mental Storage Capacity. Behavioral \& Brain Sciences, 24(1), 87-185.

[7] M Glanzer and AR Cunitz (1966). Two Storage Mechanisms in Free Recall. Journal of Verbal Learning and Verbal Behavior, 5, 351-360.

[8] A Tversky and D Kahneman (1981). The Framing of Decisions and the Psychology of Choice. Science, 211, 453-458.

[9] KA Ericsson and CA Lehmann (1996). Expert and Exceptional Performance: Evidence of Maximal Adaptation to Task Constraints. Annual Review of Psychology, 47, 273-305.

[10] E Pariser (2011). The Filter Bubble: What the Internet is Hiding From You. New York, NY: Penguin.

[11] RH Ashton and AH Ashton (1990). Evidence Responsiveness in Professional Judgment: Effects of Positive Versus Negative Evidence and Presentation Mode. Organizational Behavior and Human Decision Processes, 46, 1-19.

[12] W Yang, J Ma, H Chen, (...), M Bonaiuto, and F Babiloni (2018). Good News or Bad News, Which Do You Want First? The Importance of the Sequence and Organization of Information for Financial Decision-Making: A Neuro-Electrical Imaging Study. Frontiers in Human Neuroscience (12), 294.

[13] SR Allred, LE Crawford, S Duffy, and J Smith (2016). Working Memory and Spatial Judgements: Cognitive Load Increases the Central Tendency Bias. Psychonomic Bulletin and Review, 23(6):1825-1831.

[14] RM Hogarth and HJ Einhorn (1992). Order Effects in Belief Updating: The BeliefAdjustment Model. Cognitive Psychology, 24, 1-55. doi: 10.1016/00100285(92)90002-J

[15] BM Perrin, BJ Barnett, L Walrath, and JD Grossman (2001) Information Order and Outcome Framing: An Assessment of Judgment Bias in a Naturalistic Decision-Making Context. Human Factors, 43(2), 227-238

[16] ME Oswald and S Grosjean (2004). Confirmation Bias. In RF Pohl (Ed.) Cognitive illusions. A handbook on fallacies and biases in thinking, judgement and memory. Hove and N.Y.: Psychology Press

[17] DR Mandel (2014). Do Framing Effects Reveal Irrational Choice? Journal of Experimental Psychology: General, 143(3), 1185-1198. 